\title{
LETTER
}

Acute myeloid leukemia

\section{Restoration of microRNA function impairs MYC-dependent maintenance of MLL leukemia}

\author{
Shouhai Zhu ${ }^{1} \cdot$ Xiaoyan Cheng ${ }^{1} \cdot$ Ruiheng Wang ${ }^{1} \cdot$ Yuting $\operatorname{Tan}^{1} \cdot$ Maolin Ge $\bigodot^{1} \cdot$ Dan $\mathrm{Li}^{1} \cdot$ Qiongyu Xu ${ }^{1} \cdot$ Yan Sun ${ }^{1}$. \\ Chunjun Zhao' ${ }^{1}$ Saijuan Chen ${ }^{1} \cdot$ Han Liu $\mathbb{B}^{1}$
}

Received: 19 August 2019 / Revised: 16 January 2020 / Accepted: 12 February 2020

(c) The Author(s) 2020. This article is published with open access

\section{To the Editor:}

MicroRNAs (miRNAs) are a class of small noncoding RNAs that have critical functions in gene silencing by binding to complementary mRNAs to induce their degradation or translational repression [1]. Interestingly, only a small fraction of miRNAs can exert their function in the form of miRNA-induced silencing complex (miRISC) [2]. Unfortunately, although dysregulated expression or processing of cancer-related miRNAs has been demonstrated to play a crucial role in oncogenesis, the contribution of miRNA dysfunction is still poorly understood.

MLL-rearranged leukemias generally have a poor prognosis and account for $10 \%$ of overall acute lymphoblastic leukemia (ALL) and acute myeloid leukemia (AML) cases [3]. MLL translocations encoding chimeric fusion proteins comprising the N-terminus of MLL in frame with various fusion partner proteins are characteristically found in MLL leukemias [3]. MLL is a histone H3 Lysine4 (H3K4) methyltransferase and is proteolytically cleaved into two distinct subunits, MLL ${ }^{\mathrm{N} 320}$ and MLL ${ }^{\mathrm{C} 180}$, which noncovalently interact to form an intramolecular complex involved in epigenetic transcriptional regulation. Interestingly enough, we recently uncovered an unexpected role for

These authors contributed equally: Shouhai Zhu, Xiaoyan Cheng, Ruiheng Wang, Yuting Tan

Supplementary information The online version of this article (https:// doi.org/10.1038/s41375-020-0768-2) contains supplementary material, which is available to authorized users.

Han Liu

liuhan68@sjtu.edu.cn

1 State Key Laboratory of Medical Genomics, Shanghai Institute of Hematology, Rui Jin Hospital, School of Medicine and School of Life Sciences and Biotechnology, Shanghai Jiao Tong University, 200025 Shanghai, China
MLL protein in miRNA-mediated translational repression [4]. Our studies showed that the MLL ${ }^{\mathrm{C} 180}$ subunit alone could colocalize with miRISC components in cytoplasmic processing bodies (P-bodies), and affect the function of a subset of miRNAs such as the let-7 family [4].

Multiple miRNAs have been found to be dysregulated in MLL leukemias [5, 6]. However, since only a small fraction of miRNAs are functional, it is not clear whether the expression levels of miRNAs reflect their actual contribution to the pathogenesis of MLL leukemia. Considering that the abundance of wild-type MLL protein was reduced in MLL leukemic cells [7, 8], we reasoned that the function of a subset of miRNAs would be compromised and may play a critical role in the pathogenesis of MLL leukemia.

We first addressed how P-body formation and miRNAmediated gene silencing were affected in MLL leukemic cells. Immunofluorescence results showed that the number of DDX6- and DCP1A-marked P-bodies in MLL leukemic cells, including RS4;11, SEM, KOPN8, and THP-1 cells, was significantly fewer than in non-MLL leukemic lines such as JM1, REH, and U937 cells (Fig. 1a (i), and Supplementary Fig. S1a-c). Furthermore, the capacity of ectopically expressed let-7a or CXCR4 to silence their bulged miRNA reporters, but not perfect siRNA reporters, was markedly reduced in MLL leukemic cells (Fig. 1a (ii) and Supplementary Fig. S2a, b). These results were consistent with our previous findings that MLL was required for miRNA-mediated translational repression of partially matched mRNAs, but not for cleavage of perfectly matched mRNAs [4]. These defects in the MLL leukemic cells were associated with a reduced level of $\mathrm{MLL}^{\mathrm{C} 180}$ but not P-body proteins (Supplementary Fig. S2c). Further introduction of MLL $^{\mathrm{C} 180}$ restored miRNA-mediated gene silencing in MLL leukemic cells (Supplementary Fig. S2d), indicating that the impairment in miRNA-mediated gene silencing in MLL leukemic cells was caused by downregulating wild-type MLL, especially the MLL ${ }^{\mathrm{C} 180}$ subunit. 

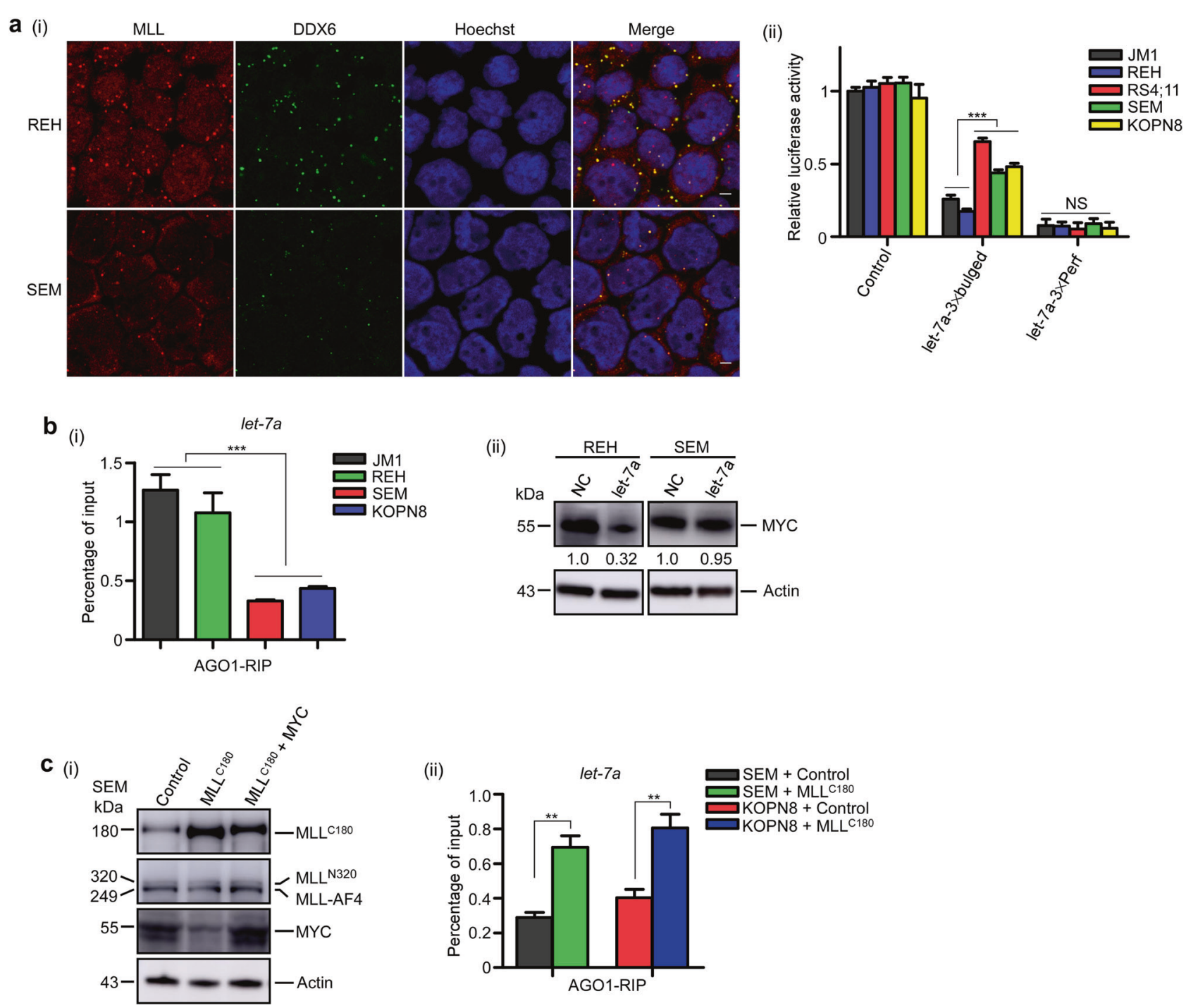

Fig. 1 MLL-fusion leukemic cells showed an impaired miRNAmediated translational repression. a (i) REH and SEM cell lines were probed with antibodies to DDX6 for immunofluorescence assay. REH cell line harbors wild-type $M L L$ gene and SEM cell line harbors $M L L-A F 4$ gene. Scale bar, $5 \mu \mathrm{m}$. (ii) The effects of MLL translocations on the function of endogenous let-7a were analyzed using dual luciferase reporter assays. The reporter activity was normalized to JM1 cells transfected with empty reporter vector. b (i) Extracts of JM1, REH, SEM, and KOPN8 cells were subjected to anti-AGO1 RIP assays. The pull-downed RNAs were analyzed by qRT-PCR using primers for let-7a. (ii) REH and SEM cells were transfected with NC

MYC represents a critical cooperation pathway and a therapeutic target in MLL-rearranged AML that is frequently upregulated in this disease $[9,10]$. We further showed that the proliferation of MLL-rearranged B-ALL cells was decreased upon MYC depletion (Supplementary Fig. S3a, b) and that MYC protein abundance in MLL-rearranged B-ALL cells was much higher than in non-MLL-rearranged B-ALL cells (Supplementary Fig. S3c), implying that both AMLand B-ALL-type MLL leukemic cells are generally dependent on high levels of MYC protein. However, although and let-7a. Proteins were detected by western blot with anti-MYC antibody at $24 \mathrm{~h}$ post transfection. c (i) SEM cells transduced with $M L L^{C 180}$, together with or without $M Y C$ were subjected to western blot assays. Antibodies were used as indicated. MLL-AF4 fusion proteins were detected using antibody specifically recognizing the amino terminus of MLL. (ii) SEM and KOPN8 cells transduced with or without $M L L^{C 180}$ were subjected to anti-AGO1 RIP assays. Pull-down RNAs were analyzed by qRT-PCR using primers for let-7a. $* P<0.05$, $* * P<$ 0.01 , $* * * P<0.001$. Data represent mean and s.e.m. of three independent experiments.

MYC is regarded as a downstream target of MLL-fusion proteins [9], the expression level of $M Y C$ mRNA was not proportionally increased (Supplementary Fig. S3c), which was also validated using publicly available microarray datasets of well-characterized primary ALL and AML patient samples (Supplementary Fig. S3d), suggesting that translational repression of $M Y C$ mRNA was reduced in MLL leukemic cells.

Since our previous study demonstrated that MLL was required for let-7a-mediated translational repression, and 
$M Y C$ is one of the most well-established let-7a targets, we reasoned that the inability of endogenous let-7a to repress translation of its target mRNAs may partly contribute to the high expression level of MYC protein in MLL leukemic cells. Therefore, we determined whether the translational repression function of endogenous let-7a was impaired in MLL leukemic cells. Since translational suppression of mRNA targets by mature miRNAs preferentially requires AGO1 [11], we performed AGO1 RNA immunoprecipitation (RIP) experiments and showed that the binding of both let-7a and MYC mRNA to AGO1 were reduced in MLL leukemic cells (Fig. 1b (i), and Supplementary Fig. S4a (i-ii), b). The pull-down assay using biotinylated let-7a further validated that the binding of AGO1 to let-7a was reduced in MLL leukemic cells (Supplementary Fig. S4c). Moreover, the protein levels of MYC decreased significantly after let-7a transfection in non-MLL-rearranged REH and JM1 cells, but not in MLL-rearranged SEM, RS4;11, and KOPN8 cells (Fig. 1b (ii) and Supplementary Fig. S4d, e). These results suggested that the expression of MYC proteins could escape translational repression by let-7a in MLL leukemic cells.

To confirm that the impaired translational repression of MYC by let-7 $a$ and the high level of MYC in MLL leukemic cells were caused by reduced $\mathrm{MLL}^{\mathrm{C} 180}$ expression, we evaluated how restoring $\mathrm{MLL}^{\mathrm{C} 180}$ affected miRNA function and MYC expression. The introduction of MLL ${ }^{\mathrm{C} 180}$ in SEM and KOPN8 cells decreased MYC expression and impaired cell proliferation, which could be recovered by MYC overexpression (Fig. 1c (i) and Supplementary Fig. S5a, b). However, the expression level of MLL-AF4 (Fig. 1c (i)) and MLL-ENL (Supplementary Fig. S5a) proteins was similar between the control and MLL-C-rescued MLL leukemic cell lines, suggesting the decreased MYC protein level in the MLL-C-rescued cells was not associated with a lower level of MLL-fusion proteins. In addition, immunofluorescence results showed that introduction of $\mathrm{MLL}^{\mathrm{C} 180}$ increased the number of P-bodies (Supplementary Fig. S5c). The binding of let-7a and MYC mRNA to AGO1 in SEM and KOPN8 cells after MLL ${ }^{\mathrm{C} 180}$ introduction was also enhanced as revealed by RIP assays (Fig. 1c (ii) and Supplementary Fig. S5d, e). These data indicated that the reduction of $\mathrm{MLL}^{\mathrm{C} 180}$ played a causal role in the miRNA functional deficiency in MLL leukemic cells.

LIN28A and LIN28B can be transcriptionally activated by MYC [9], and in turn enhance the expression of $M Y C$ by blocking the expression of let-7a [12], thus forming a regulatory circuit. Although the introduction of MLL ${ }^{\mathrm{C} 180}$ could significantly downregulate the MYC expression, it had little effect on the levels of LIN28A/LIN28B and mature let-7a (Supplementary Fig. S6a, b). Furthermore, depletion of endogenous LIN28A/LIN28B showed little effects on the binding of let-7a and $M Y C$ mRNA to AGO1
(Supplementary Fig. S6c (i-ii), d (i-ii)). These results ruled out the contribution of LIN28 in the context of $\mathrm{MLL}^{\mathrm{C} 180}$ regulated let-7a dysfunction, highlighting the dominant role of $\mathrm{MLL}^{\mathrm{C} 180}$ in controlling let-7a-mediated $M Y C$ suppression.

To further strengthen our findings, we examined whether miRNA-mediated gene silencing was impaired in primary MLL leukemic cells. Compared with the control cells, the $M L L-A F 9$-transduced primary mouse bone marrow progenitor cells showed a marked reduction in the number of P-bodies (Fig. 2a (i)). In addition, the capacity for silencing the miRNA reporters (Supplementary Fig. S7a, b) and the binding of let-7a and MYC mRNA to AGO1 (Supplementary Fig. S7c) were also significantly reduced. These defects were correlated with a high expression level of MYC protein in primary MLL leukemic cells (Supplementary Fig. S7d, e). Furthermore, ectopically expressed MLL ${ }^{\mathrm{C} 180}$ could partially rescue let-7a-mediated gene silencing in $M L L-A F 9$-transduced primary cells (Supplementary Fig. S7a, c) and significantly delay the development of leukemia in the xenografted mice (Fig. 2a (ii) and Supplementary Fig. S7f).

A previous report revealed that pharmacologically inhibiting the IRAK pathway could substantially improve survival of mice with MLL leukemia by stabilizing the wild-type MLL protein [13]. We hypothesized that IRAK inhibition could rescue the function of let-7a and decrease the expression of MYC via restoration of MLL protein levels. We first examined the effect of an IRAK1/4 inhibitor (IRAK1/4i) on miRNA-mediated gene silencing in $M L L-A F 9$-transduced primary cells. We observed that the capacity of let-7a for silencing the miRNA reporters (Supplementary Fig. S8a) and the binding of let-7a and MYC mRNA to AGO1 (Fig. 2b (i) and Supplementary Fig. S8b) were significantly increased upon IRAK1/4i treatment. In contrast, casein kinase II (CKII) inhibitor CX4945, which increases the level of full-length MLL protein by blocking taspase1-dependent MLL processing [14], had very little effect in these assays. Furthermore, treatment with IRAK1/4i, but not CX-4945, led to significant MLL $^{\mathrm{C} 180}$ induction and MYC reduction (Fig. 2b (ii) and Supplementary Fig. S8c, d). These results further support our previous finding that only free $\mathrm{MLL}^{\mathrm{C} 180}$ was implicated in miRNA-mediated translational suppression [4].

MYC represents a critical therapeutic target in MLLrearranged leukemias [10]. Bromodomain and extraterminal (BET) protein inhibitors have shown profound efficacy against MLL-rearranged leukemia by inhibiting several key targets including MYC [10]. In fact, MYC restoration constitutes one of the major mechanisms of BET inhibitor resistance [15]. We therefore reasoned that IRAK1/4i may improve the efficacy of BET inhibitors by synergistically downregulating MYC in MLL leukemias. 


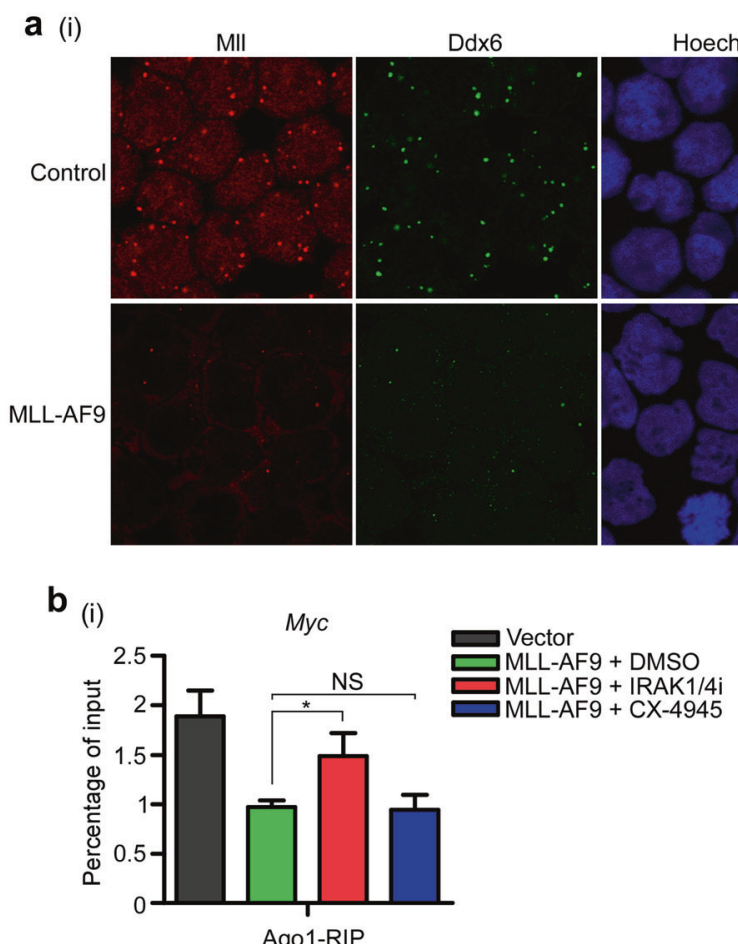

(ii)

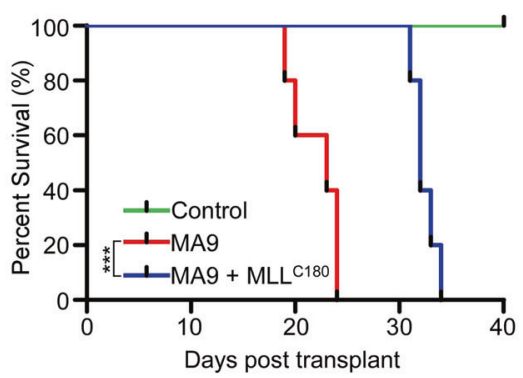

(ii)

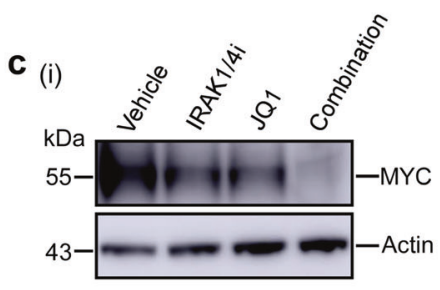

(ii) Treatment initiation at day 10

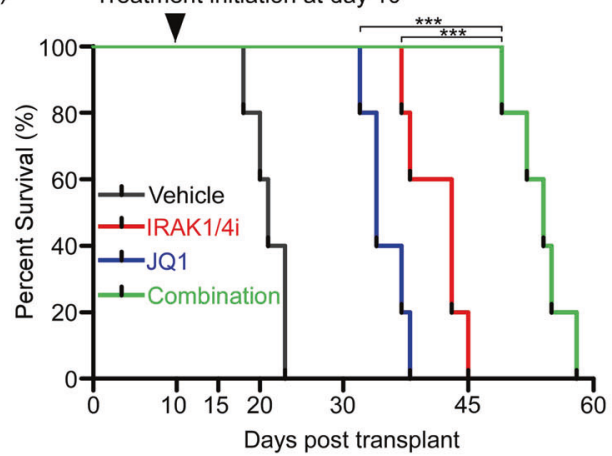

Fig. 2 Impaired miRNA-mediated translational repression in MLL-fusion leukemic cells contributes to MYC-dependent survival. a (i) Mouse bone marrow progenitor cells transduced with control or $M L L-A F 9$ gene were probed with antibodies to Ddx6 for immunofluorescence assay. Scale bar, $5 \mu \mathrm{m}$. (ii) Effect of $M L L^{C 180}$ on $M L L$ $A F 9$ mediated leukemogenesis in vivo. Kaplan-Meier survival curves were shown for three groups of normal and transplanted mice including $M L L-A F 9$ and $M L L-A F 9+M L L^{C 180}$ in a secondary bone marrow transplantation Assay ( $n=5$ per each group). b (i) Mouse bone marrow cells transduced with empty vector (control) or $M L L$ $A F 9$, were treated with DMSO (control), IRAK1/4 inhibitor $(2 \mu \mathrm{M})$, or CX-4945 $(2 \mu \mathrm{M})$ for $48 \mathrm{~h}$. Then cells were collected for the anti-Ago1 RIP assays. Pull-down RNAs were analyzed by qRT-PCR using

Indeed, in line with this hypothesis, concomitant IRAK1/4 and BET inhibition synergistically led to a significant reduction of MYC protein in MLL-AF9 primary leukemia cells (Fig. 2c (i)), and synergistically inhibited the development of MLL-rearranged leukemia in a murine xenograft model (Fig. 2c (ii) and Supplementary Fig. S8e), suggesting combination therapy with IRAK1/4 and BET inhibitors can achieve better efficacy against MLL leukemias. primers for Myc. (ii) Western blot analyses of the time-dependent effects of IRAK1/4 inhibitor treatment on MLL ${ }^{\mathrm{C} 180}$ and MYC expression in $M L L-A F 9$ primary leukemia cells. Actin was used as a loading control. c (i) $M L L-A F 9$ primary leukemia cells were treated with vehicle control, IRAK1/4 inhibitor $(2 \mu \mathrm{M})$, JQ1 $(100 \mathrm{nM})$, or combination for $24 \mathrm{~h}$. Cell lysates were collected and analyzed by western blot for the expression of MYC. (ii) Kaplan-Meier survival curves of secondary transplanted NOD-SCID mice after vehicle, JQ1, IRAK1/4 inhibitor, or combination treatment at day $10(n=5$ per each group). IRAK1/4 inhibitor ( $8 \mathrm{mg} / \mathrm{kg})$, JQ1 $(50 \mathrm{mg} / \mathrm{kg})$, or combination were administered every other day by intraperitoneal injection for a total of five treatments. $* P<0.05, * * P<0.01, * * * P<0.001$. Data represent mean and s.e.m. of three independent experiments.

In summary, our results demonstrated that the dysfunction of let-7a caused by a reduced level of MLL was essential for maintaining MYC protein at a high level and sustaining the survival of MLL leukemic cells. Thus, our work has uncovered a functional link between miRNA dysfunction and MLL-rearranged leukemia. Moreover, our work revealed that IRAK1/4 inhibition can improve the efficacy of BET inhibitors in MLL leukemias by restoring 
the protein level of MLL and the MYC-suppressing function of let-7a. We thus propose a novel rationale for synergistically targeting MYC with both IRAK and BET inhibitors as part of a comprehensive therapeutic approach for treatment of MLL leukemias.

Acknowledgements This work was supported by the National Key Research and Development Program of China (2018YFA0107802), the National Natural Science Foundation of China (81973996, 81900107, and 81570119), the Program of Shanghai Academic/Technology Research Leader (19XD1402500), the Shanghai Municipal Education Commission Gaofeng Clinical Medicine Grant (20161304), the Shanghai Municipal Health Commission (2019CXJQ01), the Shanghai Shu Guang Project (14SG15), the Shanghai Sailing Program (19YF1429500), the Collaborative Innovation Center of Hematology, and the Samuel Waxman Cancer Research Foundation.

Author contributions SHZ, XYC, RHW, and YTT designed and performed most of experiments, analyzed the data and wrote the draft paper; MLG and QYX performed some experiments; YS and DL provided technical assistance for the mouse experiments; CJZ and SJC provided expertise and extensively edited the paper; HL contributed grant support, designed the entire project, wrote the paper, and supervised the project. All authors discussed the results and commented on the paper.

\section{Compliance with ethical standards}

Conflict of interest The authors declare that they have no conflict of interest.

Publisher's note Springer Nature remains neutral with regard to jurisdictional claims in published maps and institutional affiliations.

Open Access This article is licensed under a Creative Commons Attribution 4.0 International License, which permits use, sharing, adaptation, distribution and reproduction in any medium or format, as long as you give appropriate credit to the original author(s) and the source, provide a link to the Creative Commons license, and indicate if changes were made. The images or other third party material in this article are included in the article's Creative Commons license, unless indicated otherwise in a credit line to the material. If material is not included in the article's Creative Commons license and your intended use is not permitted by statutory regulation or exceeds the permitted use, you will need to obtain permission directly from the copyright holder. To view a copy of this license, visit http://creativecommons. org/licenses/by/4.0/.

\section{References}

1. Lin S, Gregory RI. MicroRNA biogenesis pathways in cancer. Nat Rev Cancer. 2015;15:321-33.

2. Leung AK. The whereabouts of microRNA actions: cytoplasm and beyond. Trends Cell Biol. 2015;25:601-10.

3. Muntean AG, Hess JL. The pathogenesis of mixed-lineage leukemia. Annu Rev Pathol. 2012;7:283-301.

4. Zhu S, Chen Z, Wang R, Tan Y, Ge M, Sun Y, et al. MLL is required for miRNA-mediated translational repression. Cell Discov. 2019;5:43.

5. Benetatos L, Vartholomatos G. MicroRNAs mark in the MLLrearranged leukemia. Ann Hematol. 2013;92:1439-50.

6. Schotte D, De Menezes RX, Akbari Moqadam F, Khankahdani LM, Lange-Turenhout E, Chen C, et al. MicroRNA characterize genetic diversity and drug resistance in pediatric acute lymphoblastic leukemia. Haematologica. 2011;96:703-11.

7. Liu H, Westergard TD, Cashen A, Piwnica-Worms DR, Kunkle L, Vij R, et al. Proteasome inhibitors evoke latent tumor suppression programs in pro-B MLL leukemias through MLL-AF4. Cancer Cell. 2014;25:530-42.

8. Liu H, Takeda S, Kumar R, Westergard TD, Brown EJ, Pandita TK, et al. Phosphorylation of MLL by ATR is required for execution of mammalian S-phase checkpoint. Nature. 2010; $467: 343-6$.

9. Jiang X, Huang H, Li Z, Li Y, Wang X, Gurbuxani S, et al. Blockade of miR-150 maturation by MLL-fusion/MYC/LIN-28 is required for MLL-associated leukemia. Cancer Cell. 2012;22:524-35.

10. Dawson MA, Prinjha RK, Dittmann A, Giotopoulos G, Bantscheff $\mathrm{M}$, Chan WI, et al. Inhibition of BET recruitment to chromatin as an effective treatment for MLL-fusion leukaemia. Nature. 2011; 478:529-33.

11. Peters L, Meister G. Argonaute proteins: mediators of RNA silencing. Mol Cell. 2007;26:611-23.

12. Piskounova E, Polytarchou C, Thornton JE, LaPierre RJ, Pothoulakis C, Hagan JP, et al. Lin28A and Lin28B inhibit let-7 microRNA biogenesis by distinct mechanisms. Cell. 2011;147: 1066-79.

13. Liang K, Volk AG, Haug JS, Marshall SA, Woodfin AR, Bartom ET, et al. Therapeutic targeting of MLL degradation pathways in MLL-rearranged leukemia. Cell. 2017;168:59-72.e13.

14. Zhao Z, Wang L, Volk AG, Birch NW, Stoltz KL, Bartom ET, et al. Regulation of MLL/COMPASS stability through its proteolytic cleavage by taspase 1 as a possible approach for clinical therapy of leukemia. Genes Dev. 2019;33:61-74.

15. Fong CY, Gilan O, Lam EY, Rubin AF, Ftouni S, Tyler D, et al. BET inhibitor resistance emerges from leukaemia stem cells. Nature. 2015;525:538-42. 